\title{
A UNIFIED PRESENTATION OF GENERALISED VOIGT FUNCTIONS
}

\author{
M. KAMARUJJAMA ${ }^{1}$
}

(Received 10 April, 2002; revised 23 September, 2002)

\begin{abstract}
Voigt functions occur frequently in a wide variety of problems in several diverse fields of physics. This paper presents a unified study of generalised Voigt functions. In particular, some expansions of unified Voigt functions are given in terms of the original functions. Some deductions from these representations are obtained which give us an opportunity to underline the special rôle of the associated generating functions.
\end{abstract}

\section{Introduction}

The Voigt functions $K(x, y)$ and $L(x, y)$ play an important rôle in several diverse fields of physics such as astrophysical spectroscopy and the theory of neutron reactions. Furthermore, the function $K(x, y)+i L(x, y)$ is identical to the so-called 'plasma dispersion function', which has been tabulated by Fried and Conte [4].

In many given physical problems, a numerical or analytical evaluation of Voigt functions is required. As an interesting extension of the work of Reiche [6] and Exton [3], Srivastava and Miller [8] introduced and studied a unification (or generalisation) of the Voigt functions $K(x, y)$ and $L(x, y)$ in the following form:

$$
V_{\mu, v}(x, y)=\left(\frac{x}{2}\right)^{1 / 2} \int_{0}^{\infty} t^{\mu} e^{-y t-t^{2} / 4} J_{v}(x t) d t,
$$

$\left(\mu, x, y \in R^{+}\right.$and $\left.\operatorname{Re}(\mu+\nu)>-1\right)$ so that $K(x, y)=V_{1 / 2,-1 / 2}(x, y)$ and $L(x, y)=$ $V_{1 / 2,-1 / 2}(x, y)$, where $J_{v}(z)$ represents the Bessel function of order $\nu$, defined by (see [5, page 108])

$$
J_{v}(z)=\sum_{m=0}^{\infty} \frac{(-1)^{m}(z / 2)^{v+2 m}}{m ! \Gamma(v+m+1)}, \quad|z|<\infty .
$$

\footnotetext{
'Department of Applied Mathematics, Z. H. College of Engg. and Technology, Aligarh Muslim University, Aligarh-202002 (U.P.) India; e-mail: kamarujjama@ rediffmail.com.

(C) Australian Mathematical Society 2003, Serial-fee code 1446-1811/03
} 
In view of integral and series representations of generalised Voigt functions ([8] and [9]), we present here some new unified representations of generalised Voigt functions in terms of some familiar special functions of mathematical physics.

\section{Representation of generalised Voigt functions}

A well-known result, due to Watson [10, page 140], is

$$
\begin{aligned}
\left(\frac{z}{2}\right)^{\mu-\nu} J_{\nu}(a z)= & \frac{a^{\nu}}{\Gamma(1+\nu)} \sum_{m=0}^{\infty} \frac{(\mu+2 m) \Gamma(\mu+m)}{m !} \\
& \times{ }_{2} F_{1}\left[-m, \mu+m ; \nu+1 ; a^{2}\right] J_{\mu+2 m}(z),
\end{aligned}
$$

or, equivalently, that

$$
\begin{aligned}
(x t)^{(k+1) / 2} J_{v}(2 a \sqrt{x t})= & a^{\nu} \sum_{m=0}^{\infty} \frac{(\nu+k+2 m+1) \Gamma(\nu+k+m+1)}{\Gamma(\nu+m+1)} \\
& \times P_{m}^{(v, k)}\left(1-.2 a^{2}\right) J_{v+k+2 m+1}(2 \sqrt{x t}),
\end{aligned}
$$

where $\mu=\nu+k+1, z=2 \sqrt{x t}$ and $P_{n}^{(\alpha, \beta)}(x)$ denotes a Jacobi polynomial (see [5, page 254 (1)]).

On multiplying (2.2) by $\exp (t)$ and using the relation [5, page 201 (2)]

$$
e^{t} J_{v}(2 \sqrt{x t})=(x t)^{\nu / 2} \sum_{n=0}^{\infty} \frac{L_{n}^{(\nu)}(x)}{\Gamma(n+v+1)} t^{n},
$$

we obtain

$$
\begin{aligned}
e^{t}(x t)^{(k+1) / 2} J_{\nu}(2 a \sqrt{x t}) \\
=a^{v} \sum_{m=0}^{\infty} \frac{(\nu+k+2 m+1) \Gamma(\nu+k+m+1)}{\Gamma(\nu+m+1)} P_{m}^{(\nu, k)}\left(1-2 a^{2}\right) \\
\quad \times(x t)^{(\nu+k+2 m+1) / 2} \sum_{n=0}^{\infty} \frac{L_{n}^{(\nu+k+2 m+1)}(x)}{\Gamma(n+\nu+k+2 m+2)} t^{n},
\end{aligned}
$$

where $L_{n}^{(\alpha)}(x)$ denotes the Laguerre polynomial ([5, page 200]).

Now replacing $2 \sqrt{t}$ by $t$ and $x$ by $x^{2}$ respectively in (2.3), multiplying both sides by $\exp \left(-y t-t^{2} / 2\right) t^{\mu-k-1}$ and then integrating with respect to $t$ between the limits 0 and $\infty$ (see [2]), we get,

$$
V_{\mu, \nu}(a x, y)=(a x)^{\nu+1 / 2} 2^{(\mu-\nu-2) / 2} \sum_{m, n=0}^{\infty} \frac{(\nu+k+2 m+1) \Gamma(\nu+k+m+1)\left(x^{2}\right)^{m}}{\Gamma(\nu+m+1) \Gamma(\nu+k+2 m+n+2) 2^{m+n}}
$$




$$
\begin{aligned}
& \times P_{m}^{(v, k)}\left(1-2 a^{2}\right) L_{n}^{(v+k+2 m+1)}\left(x^{2}\right) \\
& \times \sum_{r=0}^{\infty} \frac{(-\sqrt{2} y)^{r}}{r !} \Gamma(\mu+\nu+2 m+2 n+r+1),
\end{aligned}
$$

where the integral (1.1) is used for the expression on the left-hand side.

Separating the $r$-series of (2.4) into its even and odd terms, we obtain

$$
\begin{aligned}
V_{\mu, \nu}(a x, y) \\
=(a x)^{v+1 / 2} 2^{(\mu-v-2) / 2} \sum_{m, n=0}^{\infty} \frac{(v+k+2 m+1) \Gamma(v+k+m+1)}{\Gamma(v+m+1) \Gamma(\nu+k+2 m+n+2)} \frac{\left(x^{2}\right)^{m}}{2^{m+n}} \\
\times P_{m}^{(v, k)}\left(1-2 a^{2}\right) L_{n}^{(\nu+k+2 m+1)}\left(x^{2}\right) \\
\quad \times\left\{\Gamma\left[\frac{\mu+\nu+1}{2}+m+n\right]{ }_{1} F_{1}\left[\frac{\mu+v+1}{2}+m+n ; \frac{1}{2} ; \frac{y^{2}}{2}\right]\right. \\
\left.-\sqrt{2} y \Gamma\left[\frac{\mu+\nu+2}{2}+m+n\right]{ }_{1} F_{1}\left[\frac{\mu+\nu+2}{2}+m+n ; \frac{3}{2} ; \frac{y^{2}}{2}\right]\right\}
\end{aligned}
$$

$\left(\operatorname{Re}(\mu+\nu)>-1, a>0\right.$ and $\left.\mu, x, y \in R^{+}\right)$.

For $a=1,(2.5)$ reduces to a new double series representation of $V_{\mu, v}(x, y)$, while the special cases $\mu=1 / 2$ and $\nu=\mp 1 / 2$ of (2.5) give representations of the Voigt functions $K(a x, y)$ and $L(a x, y)$, respectively.

\section{Expansions and generating functions}

In a similar manner, replacing $2 \sqrt{t}$ by $t$ and $x$ by $x^{2}$, respectively, in (2.2), multiplying both sides by $t^{\mu-k-1} \exp \left(-y t-t^{2} / 2\right)$ and then integrating with respect to $t$ between the limits 0 and $\infty$, we obtain the transformation

$$
\begin{aligned}
\left(\frac{x}{2}\right)^{k+1} V_{\mu, \nu}(a x, y)= & a^{\nu+1 / 2} \sum_{m=0}^{\infty} \frac{(\nu+k+2 m+1) \Gamma(\nu+k+m+1)}{\Gamma(\nu+m+1)} \\
& \times P_{m}^{(\nu, k)}\left(1-2 a^{2}\right) V_{\mu-k-1, \nu+k+2 m+1}(x, y),
\end{aligned}
$$

where the integral representation (1.1) is employed.

For $a=1,(3.1)$ reduces to

$$
\begin{aligned}
\left(\frac{x}{2}\right)^{k+1} V_{\mu, \nu}(x, y)= & \sum_{m=0}^{\infty} \frac{(\nu+k+2 m+1) \Gamma(\nu+k+m+1)(1+k)_{m}}{\Gamma(\nu+m+1) m !} \\
& \times(-1)^{m} V_{\mu-k-1, \nu+k+2 m+1}(x, y) .
\end{aligned}
$$


With the help of (2.1), we can obtain a similar expansion

$$
\begin{aligned}
\left(\frac{x}{2}\right)^{\mu-\nu} V_{\mu, \nu}(a x, y)= & a^{\nu+\frac{1}{2}} \sum_{m=0}^{\infty} \frac{(\mu+2 m) \Gamma(\mu+m)}{\Gamma(1+\nu+m)} \\
& \times P_{m}^{(\nu, \mu-\nu-1)}\left(1-2 a^{2}\right) V_{\nu, \mu+2 m}(x, y) .
\end{aligned}
$$

Now expanding both sides of (3.2) with the help of a result of Srivastava and Miller [8, page 113 (11)], we get the following expansion:

$$
\begin{aligned}
& \Gamma(\alpha) \psi_{2}\left[\alpha ; v+1,1 / 2 ;-a^{2} x^{2}, y^{2}\right] \\
& -2 y \Gamma(\alpha+1 / 2) \psi_{2}\left[\alpha+1 / 2 ; v+1,3 / 2 ;-a^{2} x^{2}, y^{2}\right] \\
& =\sum_{m=0}^{\infty} \frac{\left(x^{2}\right)^{m}}{(1+\nu)_{m}(\mu+m)_{m}} P_{m}^{(\nu, \mu-\nu-1)}\left(1-2 a^{2}\right) \\
& \quad \times\left\{\Gamma(\alpha+m) \psi_{2}\left[\alpha+m ; \mu+2 m+1,1 / 2 ;-x^{2}, y^{2}\right]\right. \\
& \left.\quad-2 y \Gamma(\alpha+1 / 2+m) \psi_{2}\left[\alpha+m+1 / 2 ; \mu+2 m+1,3 / 2 ;-x^{2}, y^{2}\right]\right\},
\end{aligned}
$$

where $\alpha=(\mu+\nu+1) / 2, \operatorname{Re}(\alpha)>0$ and $a, x, y \in R^{+}$, and $\psi_{2}$ denotes Humbert's confluent hypergeometric function of two variables, defined in [7, page 59].

Replacing $y$ by $i y(i=\sqrt{-1}$ ) in (3.3) and equating real and imaginary parts, we obtain

$$
\begin{aligned}
\psi_{2}[\alpha ; \nu & \left.+1,1 / 2 ;-a^{2} x^{2},-y^{2}\right] \\
= & \sum_{m=0}^{\infty} \frac{(\alpha)_{m}\left(x^{2}\right)^{m}}{(1+\nu)_{m}(\mu+m)_{m}} P_{m}^{(\nu, \mu-\nu-1)}\left(1-2 a^{2}\right) \\
& \times \psi_{2}\left[\alpha+m ; \mu+2 m+1,1 / 2 ;-x^{2},-y^{2}\right]
\end{aligned}
$$

Finally, taking $y=0$ in (3.4), using a relation from [8, page 254 (1)] and adjusting the variables, we get the Erdélyi multiplication formula [1, page 283 (7)], (see also [7, page $236(55)])$ :

$$
\begin{aligned}
{ }_{1} F_{1}[\alpha ; v+1 ; a x]= & \sum_{m=0}^{\infty} \frac{(\alpha)_{m}}{(\mu+m)_{m}} \frac{(-x)^{m}}{m !}{ }_{2} F_{1}\left[\begin{array}{cc}
-m, \mu+m & ; \\
v+1 & ;
\end{array}\right] \\
& \times{ }_{1} F_{1}\left[\begin{array}{cc}
\alpha+m & ; \\
\mu+2 m+1 ;
\end{array}\right],
\end{aligned}
$$

where the parameter $\mu$ is so restricted that the second expression exists. 


\section{Acknowledgements}

I am thankful to the referee and to Prof. M. A. Pathan of Aligarh Muslim University, Aligarh (India) for their valuable suggestions which led to an improvement of this paper.

\section{References}

[1] A. Erdélyi, W. Magnus, F. Oberhettinger and F. G. Tricomi, Higher transcendental functions, Volume 1 (McGraw-Hill, New York, 1953).

[2] A. Erdélyi, W. Magnus, F. Oberhettinger and F. G. Tricomi, Table of integral transforms, Volume 1 (McGraw-Hill, New York, 1954).

[3] H. Exton, "On the reducibility of the Voigt functions", J. Phys. A.: Math. Gen. 14(1981) L75-L77.

[4] B. D. Fried and S. D. Conte, The plasma dispersion function (Academic Press, New York, 1961).

[5] E. D. Rainville, Special functions (The Macmillan Comp., New York, 1960).

[6] F. Reiche, "Uber die Emission, Absorption and Intesitatsverteilung von Spektrallinien", Ber. Deutsch Phys. Ges. 15 (1913) 3-21.

[7] H. M. Srivastava and H. L. Manocha, A treatise on generating functions (Halsted Press, New York, 1984).

[8] H. M. Srivastava and E. A. Miller, "A unified presentation of the Voigt functions", Astrophys. Space Sci. 135 (1987) 111-118.

[9] H. M. Srivastava, M. A. Pathan and M. Kamarujjama, "Some unified presentations of the generalized Voigt functions", Comm. Appl. Anal. 2 (1998) 49-64.

[10] G. N. Watson, A treatise on the theory of Bessel functions (Cambridge Univ. Press, Cambridge, 1944). 